\title{
LOW POWER ELECTROSTATIC HELMHOLTZ-RESONANCE MICROJET GENERATOR FOR PROPULSION AND COOLING
}

\author{
Hanseup Kim ${ }^{1}$, Abel H. Jauregui ${ }^{2}$, Christian Morrison ${ }^{1}$, \\ Khalil Najafi $^{1}$, Luis P. Bernal ${ }^{1}$, and Peter D. Washabaugh ${ }^{1}$
}

\author{
${ }^{1}$ Center for Wireless Integrated MicroSystems (WIMS ERC), University of Michigan, Ann Arbor, MI \\ ${ }^{2}$ Center for Wireless Integrated MicroSystems, New Mexico Institute of Mining and Technology, Socorro, NM
}

\begin{abstract}
This paper presents the fabrication and test results of an electrostatic Helmholtz-resonance micro-acoustic actuator for high-velocity air jet generation. It incorporates a curvedelectrode electrostatic actuator $(\sim 8.8 \mu \mathrm{m}$ deep $)$ to provide twice better performance than previously reported devices that used a flat-electrode design. The out-of-plane curved electrode is formed on a silicon wafer through the buckling of stressed thin films forming the electrode.

The fabricated device has a footprint of $1.6 \times 1.6 \mathrm{~cm}^{2}$ and contains 25 acoustic micro thrusters. It operates using a $140 \mathrm{~V}$ and $70 \mathrm{kHz}$ sinusoidal signal and produces: 1) thrust of $55.6 \mu \mathrm{N}$, 2) maximum air velocity of $1.2 \mathrm{~m} / \mathrm{s}$, and 3) average velocity of $1.0 \mathrm{~m} / \mathrm{s}$ across the whole chip. The average power consumption of the 25 micro thruster array is $3.11 \mathrm{~mW}$. The generated jet was visualized by pumping ethanol clouds into a vertical gas stream up to $12 \mathrm{~cm}$. In addition, the fabricated microjets successfully cooled a heated $\left(\sim 51^{\circ} \mathrm{C}\right)$ aluminum plate $(1.6 \times 1.6$ $\mathrm{cm}^{2}$ ) down by $\sim 10^{\circ} \mathrm{C}$, which corresponds to a cooling power of $1138 \mathrm{~W} / \mathrm{m}^{2}$ per unit area at a temperature difference of $100^{\circ} \mathrm{C}$.
\end{abstract}

Key Words: Buckled Electrode, Electrostatic actuator, Helmholtz resonance, Micro jet and propulsion.

\section{INTRODUCTION}

Microjets are useful in many applications, including chip cooling, gas pumping, and air-breathing micro thrusters for propulsion. To achieve high air velocity (and therefore high thrust), high-frequency and large-volume displacement of an actuation membrane at Helmholtz resonance is desirable (Figure 2) [1]. However, in electrostatic actuators, large-volume displacement is limited by the small gap between a diaphragm and an electrode. The small gap is necessary to keep the operation voltage low enough for practical electrostatic actuation. Such limitation can be overcome by using a curved electrode electrostatic actuator [2]. The curved electrode keeps the gap small between a membrane and a diaphragm around the peripheral, but allows a large deflection at the center. Thus, it achieves a large volume displacement at a low voltage.

Out-of-plane curved electrode structures can be easily microfabricated by engineering the buckling of stressed thin films [3]. This buckling method is simpler, cheaper, and more uniform than other methods, such as DRIE lag [4] and grayscale photo-mask sets [5].

In this paper, we report a Helmholtz-resonance microjet generator with large-volume displacement and low power consumption, using a buckled curved electrode design. Its jetting and propulsion performance as well as cooling capability are also reported.

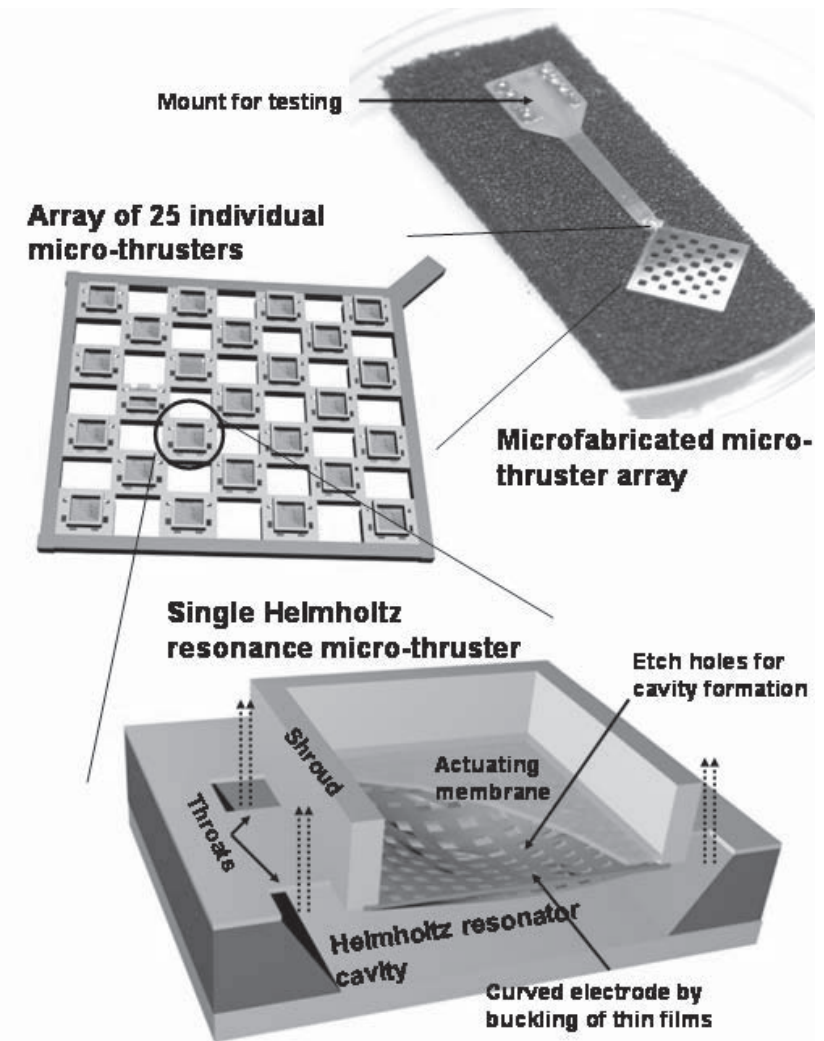

Figure 1. Conceptual illustration of a single micro-thruster with a curved electrode (bottom), array of 25 micro-acoustic-thrusters (center), and the fabricated micro-thruster array that is mounted on a $P C$ board (top).

\section{LOW-POWER HELMHOLTZ MICROJET}

\section{Helmholtz resonance microject}

Figure 2 shows the operation of a Helmholtz-resonance microjet generator. When a membrane is deflected by a buckled electrode to compress the air inside a Helmholtz-resonance cavity, a directional air jet is ejected from the cavity (Figure 2. (a)). Since the ejected jet is directional, it transfers a large momentum out in the vertical direction. When the membrane returns to its original position due to its restoring force, the cavity volume is expanded and air is pulled into it from outside (Figure 2. (b)). The inhaled air comes from all directions and thus produces only a small momentum vertically into the cavity. Such single deflection and restoration of the membrane completes a cycle, and repeats at a high-frequency resulting in a net momentum generated and zero mass transfer per cycle. The operation frequency is determined by the geometry of the cavity. 

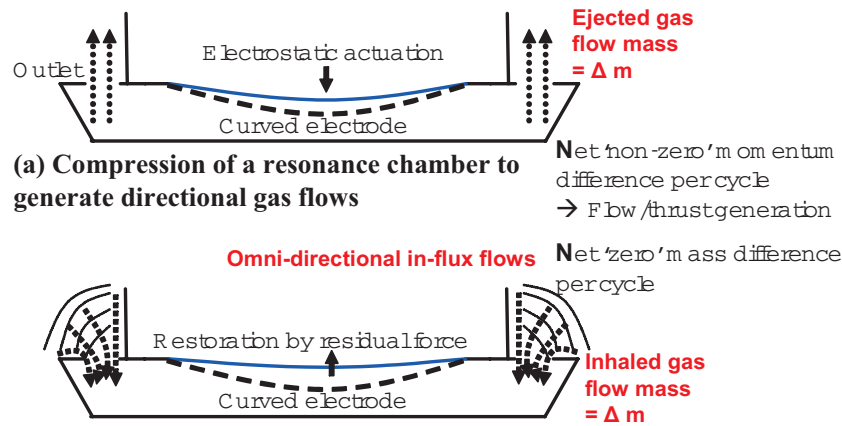

(b) Expansion of a resonance chamber to inhale omni-directional gas flows

Figure 2. Operation principle of the electrostatic acoustic microjet generator.

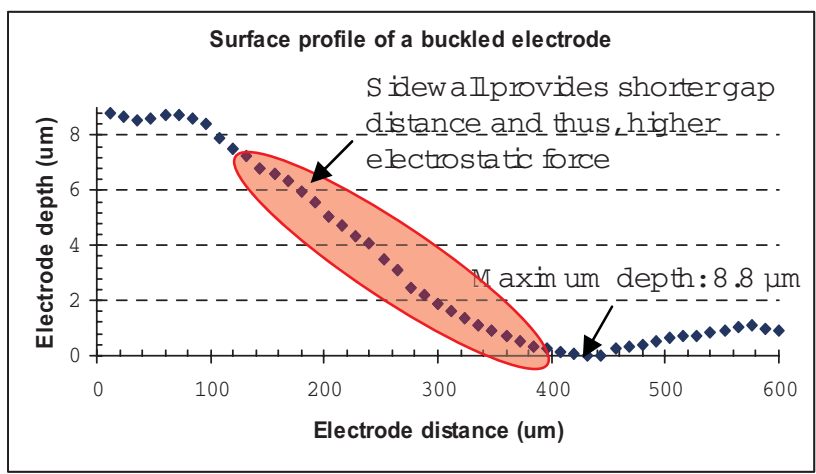

Figure 3. Surface profile of the fabricated curved electrode over half the whole electrode. This curvature enables low-voltage electrostatic operation by reducing the gap distance around the periphery while maintaining a large separation in the center of the actuator [3].

\section{Buckled electrode electrostatic actuator}

Curved electrodes are built by utilizing the intrinsic stress in thin films. Thin films are deposited on a substrate and then released from the substrate to form a suspended diaphragm. The built-in compressive stress in these thin films causes buckling out of the plane of the wafer, thus forming a naturally0curved diaphragm, which can be used a s curved electrode., as shown in Figure 3 [3]. The buckled electrode provides a smooth curvature around the periphery, which is necessary for lowvoltage operation. In addition, it also provides high structural strength, preventing the movement of electrodes during electrostatic attraction [3].

\section{FABRICATION}

Figure 4 shows a simplified microfabrication process flow. First, layers of compressively stressed thermally grown silicon oxide $(0.5-\mu \mathrm{m})$ and LPCVD polysilicon $(3.8-\mu \mathrm{m})$ were deposited. Then, the polysilicon layer was doped with boron to provide high resistance against the TMAH wet etching during the cavity formation. On top of the doped polysilicon, a layer of tensile silicon nitride $(0.1-\mu \mathrm{m})$ was deposited to induce the electrode to buckle downwards [6]. The composite layers were patterned with perforation holes using DRIE etching. These perforation holes were used to wet-etch the silicon substrate (undoped) under the composite membrane and also to reduce

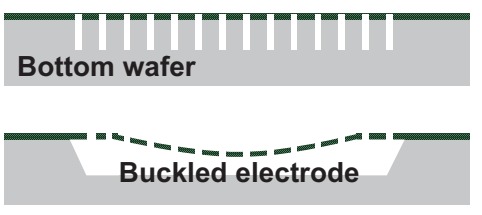

Boron-doping/D R $\mathbb{E}$ etching forwetetch access

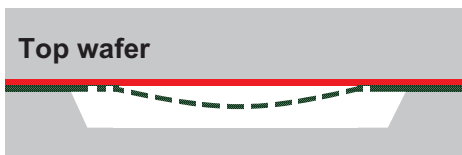

$W$ aferbonding using a polym er, BCB [2]

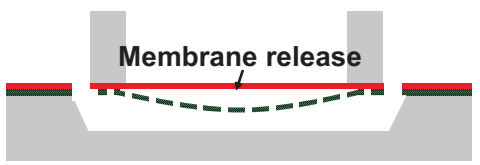

D R $\mathbb{E}$ bu $\mathbb{k}$ silicon etching form em brane form ation

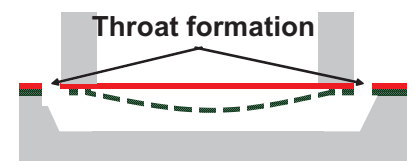

D R $\mathbb{E}$ silicon etching for device separation
Figure 4. Simplified microfabrication process

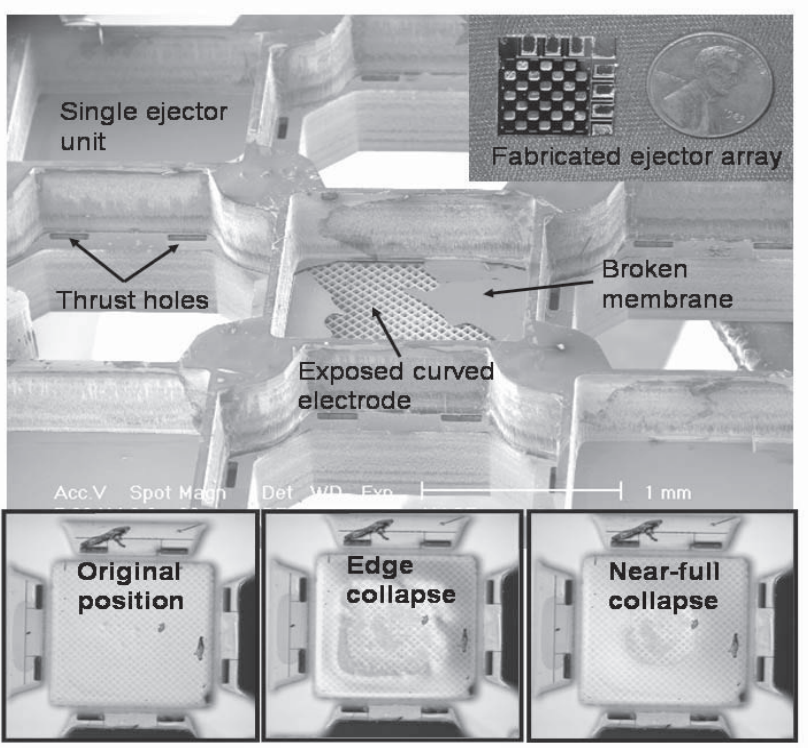

Figure 5. (Top) SEM view of a fabricated micro actuator array. Individual units have two holes per side (total eight thrust holes per device). (Bottom) Sequential photos of membrane collapse by a curved electrode.

damping during membrane actuation. When the silicon substrate was etched, the composite layers were buckled due to the intrinsic compressive stress. Then, a second wafer deposited with several films forming a moveable diaphragm was bonded using BCB [1]. Finally, the second wafer DRIE etched to release the moveable diaphragm, and to form microjet throats (holes).

The fabricated device contains 25 individual microjet generators in a chess-board array, as shown in Figure 5. Each individual microjet generator has an actuation membrane at the center and eight throats on four sides. Under the membrane, a perforated curved electrode is located over a Helmholtz-resonance cavity. The curved electrode gradually deflects the membrane down from the edges toward the center, as shown in Figure 5. 


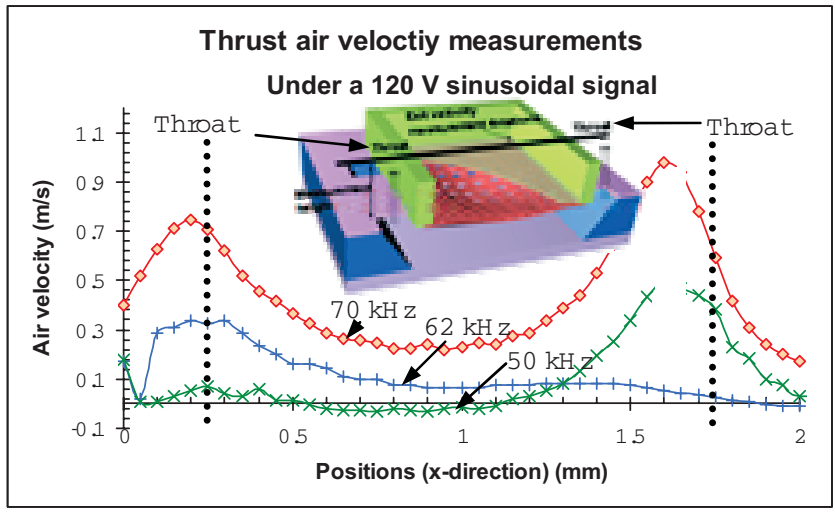

Fig 6. Air velocity measurements across an individual microthruster (refer to the $3 D$ drawing) at different frequencies. Thrust values are highest over the thrust holes (throats). Thrust generation shows a maximum value at a resonance frequency of $70 \mathrm{kHz}$.

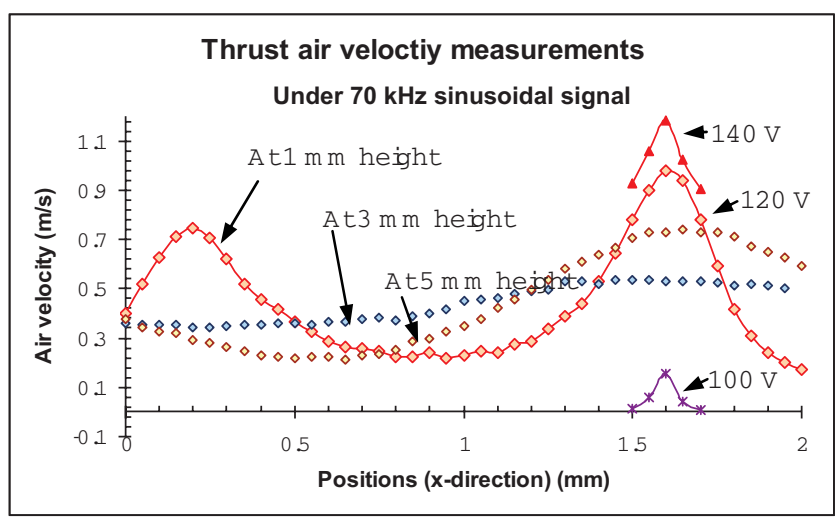

Fig 7. Air velocity measurements across an individual microthruster at different heights and voltages.

\section{TEST RESULTS}

The fabricated microjet generators (Figure 5) were first tested to determine the resonant frequency using a microphone located 2-cm above the chip. Peaks in the acoustic spectrum identify the resonant frequencies. Next, the air flow velocity produced by the micro-thrusters, actuated by a $140 \mathrm{~V}$ sinusoidal signal at $70 \mathrm{kHz}$, was measured using a hot-wire anemometer, which was placed at various heights above the chip and scanned across the whole chip to determine the variation in flow velocity. The jet flow was visualized with ethanol droplets formed by a commercial nebulizer. The thrust was also measured directly using a pendulum balance. Finally, cooling capability was verified by measuring temperature variations of a target surface when cooled using generated air jet.

\section{Hot-wire anemometry test (microjet measurement)}

The hot-wire anemometry tests were performed to directly measure the velocity from a microjet. A hot-wire (TSI model $1210 \mathrm{~T} 1.5)$ was attached to a micro-manipulator with three-axis freedom and then placed on various locations across an individual microjet generator, while its resistance variation is monitored. The specific hot-wire test setup was calibrated through a series of wind-tunnel test to attune the relationship

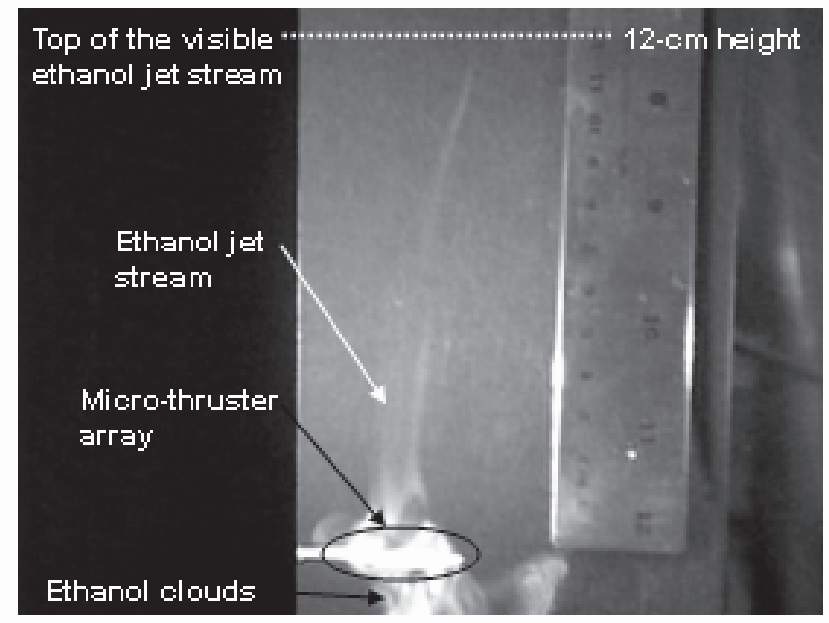

Fig 8. Visualization of thrust generation (video clips): Ethanol jetting test results show that an ethanol column was generated vertically more than $12 \mathrm{~cm}$ above the chip and ethanol clouds.

between resistance variation and air velocity. The hot-wire test was also performed under various operation voltages and at different heights.

Test results (Figures 6,7 ) show that the fabricated individual microjet generator produced a maximum air velocity of $\sim 1.2 \mathrm{~m} / \mathrm{s}$, and an average velocity of $\sim 1.0 \mathrm{~m} / \mathrm{s}$ across the device, when the velocity is measured at $1-\mathrm{mm}$ above the throat. The measured air velocity is maximized at a resonance frequency of $72 \mathrm{kHz}$, a voltage of $140 \mathrm{~V}$, and a location close to the throat (1-mm high).

\section{Jetting test (microjet visualization)}

To visualize the jetting performance, a fabricated device was actuated to jet an ethanol cloud. The ethanol cloud was formed using a commercial nebulizer under a microjet generator array, and then jetted through the thruster when it was turned on. The jetting tests (Figure 8) show that the microjet generators can pump an ethanol aerosol into a column up to $12 \mathrm{~cm}$.

\section{Thrust performance}

To measure the thrust generated by a microjet array, the microjet generator array (total mass $2.0 \mathrm{~g}$ including mounts) was attached to a $1.8-\mathrm{m}$ long string balance and operated at $70 \mathrm{kHz}$ with a $140 \mathrm{~V}$ sinusoidal driving signal. Then, the moving distance was measured to calculate the generated thrust.

Test results (Figure 10, bottom) show that the microjet array (25 devices) traveled over a distance of $4.5 \mathrm{~mm}$, which corresponds to a thrust of $55.6 \mu \mathrm{N}$. This measured thrust value corresponds to 2.2 $\mu \mathrm{N}$ per microjet generator. The measured thrust is at least twice higher than the thrust of $1.08 \mu \mathrm{N}$ from the previous microjet generators with flat electrodes [1].

To further illustrate thrust performance, the microjet device was operated in the pendulum balance at the same driving signal of $140 \mathrm{~V}, 70 \mathrm{kHz}$ and modulated at $0.43 \mathrm{~Hz}$, the resonant frequency of the pendulum. The resulting oscillatory motion is illustrated in Figure 10. The maximum displacement amplitude was $8.3 \mathrm{~cm}$.

\section{Cooling performance}

Finally, cooling capability was measured with the device generating air jets toward a target (heated) plate at a distance of $4 \mathrm{~mm}$. The target consists of an aluminum plate $\left(25 \times 25 \times 0.5 \mathrm{~mm}^{3}\right)$ attached to a heating element (Omega model KHLV-101/10) with the 

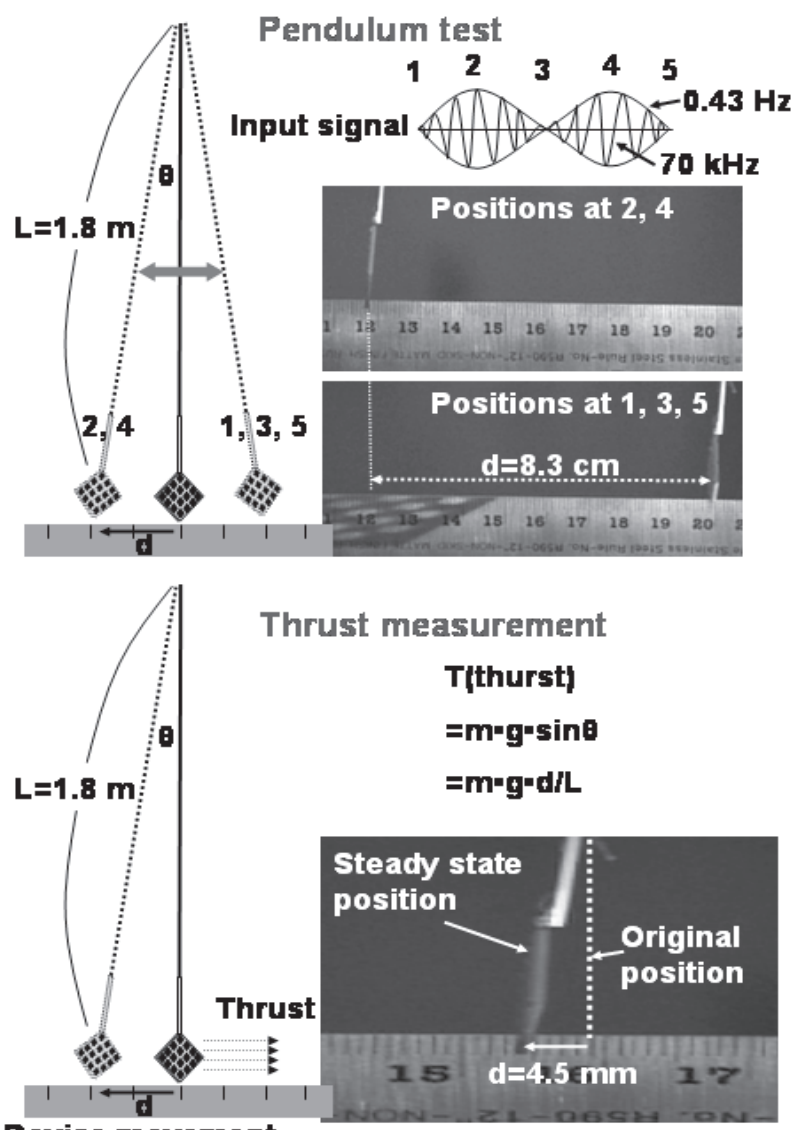

\section{Device movement}

Fig 10. Visualization of thrust generation (video clips): (Top) Pendulum test show the micro device travels $\sim 8.3-\mathrm{cm}$ distance back and forth by a $0.43-\mathrm{Hz}$ amplitude modulation of the sinusoidal $70 \mathrm{kHz}$ driving signal under $140 \mathrm{~V}$. (Bottom) Thrust measurement show the device moved 4.5-mm under the continuous application of a $140 \mathrm{~V}, 70 \mathrm{kHz}$ ac drive signal, this deflection corresponds to a thrust of $55 \mu N$.

same size. The temperature variations over the heating element were measured at three different locations by using three type-K thermocouples (Omega SC-GG-K-30-36-PP) and a digital thermometer (Omega HH501DK).

Figure 9 summarizes the temperature difference from ambient and cooling power per unit area when the array is on and off. As can be seen, a heated aluminum plate was cooled down from $\sim 51{ }^{\circ} \mathrm{C}$ to $\sim 41^{\circ} \mathrm{C}$. This cooling power can be estimated to $1138 \mathrm{~W} / \mathrm{m}^{2}$ per unit area at a temperature difference of $100^{\circ} \mathrm{C}$, which is higher than the cooling power of $600 \mathrm{~W} / \mathrm{m}^{2}$ from the previous flat-electrode design [7].

\section{DISCUSSIONS AND CONCLUSIONS}

The fabricated microjet generator with a buckled electrode has demonstrated that it can achieve a higher performance at lower-power consumption than the previous flat-electrode design, as summarized in Table 1. It improved the microjet thrust by $107 \%$ and the cooling power by $90 \%$, while operation voltage is increased from $120 \mathrm{~V}$ to $140 \mathrm{~V}$.

A few notable observations were also made during testing. First, the curved electrode was not able to deflect the membrane completely at the center. This inability came from the relationship between the increasing restoration force of a stretched membrane and the decreasing attractive force from a

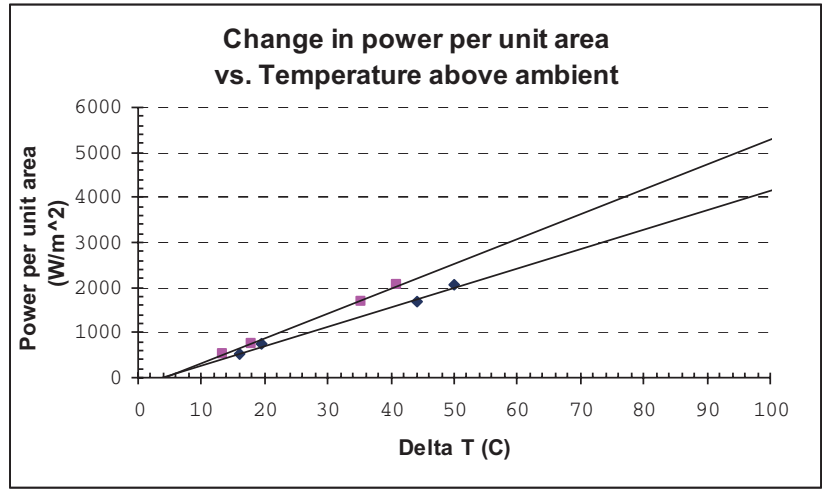

Fig 9. Cooling test results show that the generated air jet can be used to cool down a heated surface. The jet is directed toward the target surface.(pink square: after cooling, blue diamond: before cooling).

Table 1: Performance summary.

\begin{tabular}{l|l|l}
\hline Specification & $\begin{array}{l}\text { Curved electrode } \\
\text { micro ejector }\end{array}$ & $\begin{array}{l}\text { Flat electrode } \\
\text { micro ejector [1] }\end{array}$ \\
\hline $\begin{array}{l}\text { Diaphragm } \\
\text { deflection }\end{array}$ & $\mathbf{8 . 8} \boldsymbol{\mu m}$ & $4 \mu \mathrm{m}$ \\
Thrust & $\mathbf{5 5 . 6} \boldsymbol{\mu N}$ & $26.9 \mu \mathrm{N}$ \\
Max. velocity & $1.2 \mathrm{~m} / \mathrm{s}$ & $0.80 \mathrm{~m} / \mathrm{s}$ \\
Average velocity & $1.0 \mathrm{~m} / \mathrm{s}$ & $0.35 \mathrm{~m} / \mathrm{s}$ \\
Dimension $\left(\mathrm{cm}^{3}\right)$ & $1.6 \times 1.6 \times 0.1$ & $1.6 \times 1.6 \times 0.1$ \\
Voltage & $140 \mathrm{~V}$ & $120 \mathrm{~V}$ \\
\hline
\end{tabular}

reduced electrode area. This implies that another type of a curved electrode could be more efficient. Second, a higher applied voltage still could generate a higher performance. To protect the fabricated device, voltages higher than $140 \mathrm{~V}$ were not applied.

\section{ACKNOWLEDGEMENT}

The authors thank Fernando Cheng for his help on the hot-wire measurement testing set-up. This project is partially funded by the Engineering Research Centers Program of the National Science Foundation under Award Number EEC-9986866.

\section{REFERENCES}

[1] T.-K. A. Chou, K. Najafi, M. O. Muller, L. P. Bernal, and P. D. Washabaugh, "High-density micromachined acoustic ejector array for micro propulsion," Transducers '01, pp. 890-893, 2001.

[2] Legtenberg-R, Gilbert-J, Senturia-SD, and Elwenspoek-M, "Electrostatic curved electrode actuators," Journal-ofMicroelectromechanical-Systems, vol. 6, no.3, pp.257-265, 1997.

[3] H. Kim, K. Najafi, P. D. Washabaugh, and L. P. Bernal, "Largedeflection out-of-plane electrostatic buckled-electrode actuator," Transducers '03, pp. 794-797, 2003.

[4] Chou-T-KA and Najafi-K, "Fabrication of out-of-plane curved surfaces in Si by utilizing RIE lag," MEMS '02, pp.145-8, 2002.

[5] Gimkiewicz-C, Hagedorn-D, Jahns-J, Kley-E-B, and Thoma-F, "Fabrication of microprisms for planar optical interconnections by use of analog gray-scale lithography with high-energy-beamsensitive glass," Applied-Optics, vol.38, no.14, pp.2986-90, 1999.

[6] L.-S. Fan, R. S. Muller, W. Yun, R. T. Howe, and J. Huang, "Spiral microstructures for the measurement of average straingradients in thin films," MEMS '90, pp. 177-181, 1990.

[7] T.-K. A. Chou, K. Najafi, M. O. Muller, L. P. Bernal, P. D. Washabaugh, and B. A. Parviz, "Micromachined e-jet for IC chip cooling," ISSCC '02, pp. 356-357, 2002. 